\title{
STABILIZATION OF SOLUTIONS OF WEAKLY SINGULAR QUENCHING PROBLEMS
}

\author{
MAREK FILA, HOWARD A. LEVINE, AND JUAN L. VAZQUEZ
}

(Communicated by Barbara L. Keyfitz)

ABstract. In this paper we prove that if $0<\beta<1, D \subset R^{N}$ is bounded, and $\lambda>0$, then every element of the $\omega$-limit set of weak solutions of

$$
\begin{gathered}
u_{t}-\Delta u+\lambda u^{-\beta} \chi_{u>0}=0 \quad \text { in } D \times[0, \infty), \\
u= \begin{cases}1 & \text { on } \partial D \times(0, \infty), \\
u_{0}>0 & \text { on } \bar{D} \times\{0\}\end{cases}
\end{gathered}
$$

is a weak stationary solution of this problem. A consequence of this is that if $D$ is a ball, $\lambda$ is sufficiently small, and $u_{0}$ is a radial, then the set $\{(x, t) \mid u=0\}$ is a bounded subset of $D \times[0, \infty)$.

We consider the problem

$$
\begin{array}{ll}
u_{t}=\Delta u-\lambda u^{-\beta} \chi_{u>0}, & x \in D, t>0 ; \\
u=1, & x \in \partial D, t>0 ; \\
u(\cdot, 0)=u_{0}>0, & x \in \bar{D},
\end{array}
$$

with $\lambda>0,0<\beta<1$. We assume that $D$ is a bounded domain in $\mathbb{R}^{N}$ and that $u_{0}$ and $\partial D$ are of class $C^{2+\alpha}, u_{0}=1$ on $\partial D$.

This problem can be considered as a limiting case of models in chemical catalyst kinetics (Langmuir-Hinshelwood model) or of models in enzyme kinetics; see $[A, D]$.

It is known that there are initial functions $u_{0}$ for which the corresponding solutions reach zero (quench) in finite time; see [L]. Let $a \in D$ and $0<T<\infty$ be such that $u>0$ in $\bar{D} \times[0, T)$ and $u(a, T)=0$. Under some additional assumptions on $u_{0}$ and $D$, all terms in the equation blow up as $t \rightarrow T$ (for blow up of $u_{t}$ see, e.g., [DL]; for blow up of $\Delta u$ see [FK]). This means that the solution ceases to exist in the classical sense as $t \rightarrow T$. On the other hand, it was shown in [Ph] (see also [BB]) that weak solutions exist globally. They can be obtained as limits of solutions of regularized problems, they are continuous, and the gradient (with respect to $x$ ) is continuous (see $[\mathrm{Ph}]$ ). Uniqueness of

Received by the editors Feburary 26, 1992.

1991 Mathematics Subject Classification. Primary 35K55.

The second author was supported in part by NSF Grant DMS 8822788. This work was supported in part by the Institute for Mathematics and its Applications. 
the weak solutions is an open question. By a solution we mean the (unique) maximal weak solution obtained via regularization.

In this paper we are interested in the behavior of solutions as $t \rightarrow \infty$. It follows from [Ph, Lemma 5] that every orbit is relatively compact in $C(\bar{D})$. In other words, the $\omega$-limit set

$$
\begin{aligned}
& \omega\left(u_{0}\right):=\left\{v \in W^{1,2}(D) \cap C(\bar{D}):\right. \text { there is a }\left\{t_{n}\right\}, t_{n} \rightarrow \infty, \text { such that } \\
&\left.u\left(\cdot, t_{n} ; u_{0}\right) \rightarrow v \text { uniformly as } n \rightarrow \infty\right\}
\end{aligned}
$$

is nonempty. We show that $\omega\left(u_{0}\right)$ consists of (weak) stationary solutions.

For a description of the set of stationary solutions see, e.g., [JL, BN]; for the stability properties see [L] in the case $N=1$ and [FHQ] in the case $N>1, D$ a ball.

One consequence of our result is the eventual positivity of any solution if $D$ is a ball and $\lambda$ is small enough. More precisely, for any radially symmetric $u_{0}$ there is a $\tau\left(u_{0}\right) \geq 0$ such that $u\left(x, t ; u_{0}\right)>0$ on $\bar{D} \times\left(\tau\left(u_{0}\right), \infty\right)$. Hence, the frozen zone (dead core, quenching set) $u=0$, where no reaction takes place, disappears. This follows from the fact that there is a unique positive stationary solution if $D$ is a ball and $\lambda$ is small enough.

On the other hand, if $D$ is a ball and $\lambda$ is large enough, then all solutions converge (in the sup-norm) to the unique (nonclassical) stationary solution with a zero set of positive measure.

This shows that the conjecture from [L, §5] is true for large intervals and false for small intervals.

The notation

$$
f(u)=u^{-\beta} \chi_{u>0}, \quad f_{\varepsilon}(u)=\frac{u}{\varepsilon+u^{\beta+1}}
$$

will be used throughout the paper.

Lemma 1. Let $\left\{t_{n}\right\}$ be a sequence for which $u\left(\cdot, t_{n} ; u_{0}\right)$ converges in $L^{2}(D)$. If $v$ is the limit element and $U_{n}(x, s):=u\left(x, t_{n}+s ; u_{0}\right), s \in[0,1]$, then $U_{n} \rightarrow v$ in $L^{2}(D \times(0,1))$.

Proof. For the reader's convenience we prove first that

$$
\int_{0}^{\infty} \int_{D}\left(u_{t}\right)^{2} d x d t<\infty .
$$

Although not explicitly stated, this was in fact shown in [Ph, BB]. Let $u_{\varepsilon}$ be the solution of

$$
\begin{array}{ll}
u_{\varepsilon t}=\Delta u_{\varepsilon}-\lambda f_{\varepsilon}\left(u_{\varepsilon}\right), & x \in D, t>0 ; \\
u_{\varepsilon}=1, & x \in \partial D, t>0 ; \\
u_{\varepsilon}(\cdot, 0)=u_{0} & x \in \bar{D} .
\end{array}
$$

Then the following identity holds for $T>0$ :

$$
\begin{gathered}
\int_{0}^{T} \int_{D}\left(u_{\varepsilon t}\right)^{2} d x d t+J_{\varepsilon}\left(u_{\varepsilon}(\cdot, T)\right)=J_{\varepsilon}\left(u_{0}\right), \\
J_{\varepsilon}(w):=\frac{1}{2} \int_{D}|\nabla w|^{2} d x+\lambda \int_{D} \int_{0}^{2} f_{\varepsilon}(u) d u d x .
\end{gathered}
$$


Obviously, $J_{\varepsilon}(w) \geq 0$ and

$$
J_{\varepsilon}\left(u_{0}\right) \leq J\left(u_{0}\right):=\frac{1}{2} \int_{D}\left|\nabla u_{0}\right|^{2} d x+\frac{\lambda}{1-\beta} \int_{D} u_{0}^{1-\beta} d x,
$$

therefore

$$
\int_{0}^{T} \int_{D}\left(u_{\varepsilon t}\right)^{2} d x d t \leq J\left(u_{0}\right)
$$

hence, $u_{\varepsilon t}$ is weakly convergent in $L^{2}(D \times(0, T))$. Since $u_{\varepsilon} \rightarrow u$ pointwise as $\varepsilon \rightarrow 0$, the weak limit of $u_{\varepsilon t}$ is $u_{t}$, which yields $\int_{0}^{T} \int_{D}\left(u_{t}\right)^{2} d x d t \leq J\left(u_{0}\right)$ for $T>0$, and (1) follows.

Now we can finish the proof of the lemma using an idea from [LP]:

$$
\int_{D}\left|u\left(x, t_{n}+s\right)-u\left(x, t_{n}\right)\right|^{2} d x \leq \int_{D} \int_{t_{n}}^{t_{n}+1}\left(u_{t}\right)^{2} d x d t
$$

hence,

$$
\left\|U_{n}-u\left(\cdot, t_{n}\right)\right\|_{L^{2}(D \times(0,1))}^{2} \leq \int_{t_{n}}^{\infty} \int_{D}\left(u_{t}\right)^{2} d x d t .
$$

According to (1), the right-hand side goes to zero as $n \rightarrow \infty$.

Lemma 2. If $v \in \omega\left(u_{0}\right)$ then $\int_{D} f(v) \psi d x<\infty$ for any $\psi \in C^{2}(\bar{D})$ vanishing on $\partial D, \psi \geq 0$.

Proof. Choose $\rho \in C_{0}^{2}((0,1)), \rho \geq 0, \int_{0}^{1} \rho(s) d s=1$, and set $g(x, t)=$ $\psi(x) \rho\left(t+t_{n}\right)$. Then

$$
\int_{t_{n}}^{t_{n}+1} \int_{D} u\left(\Delta g+g_{t}\right) d x d t-\lambda \int_{t_{n}}^{t_{n}+1} \int_{D} f(u) g d x d t-\int_{\partial D} \frac{\partial \psi}{\partial \nu} d S=0,
$$

and the substitution $t-t_{n}=s$ leads to

$$
\lambda \int_{0}^{1} \int_{D} f\left(U_{n}\right) g d x d t=\int_{0}^{1} \int_{D} U_{n}\left(\Delta g+g_{t}\right) d x d t-\int_{\partial D} \frac{\partial \psi}{\partial \nu} d S .
$$

Hence, there is a constant $K$ (which depends only on $g$ ) such that

$$
\int_{0}^{1} \int_{D} f\left(U_{n}\right) g d x d t \leq K \quad \text { for any } n .
$$

From Lemma 1 we obtain the existence of a subsequence (denoted again by $\left.U_{n}\right)$ such that $f_{\varepsilon}\left(U_{n}\right) \rightarrow f_{\varepsilon}(v)$ pointwise in $D \times(0,1)$; therefore,

$$
\int_{0}^{1} \int_{D} f_{\varepsilon}\left(U_{n}\right) g d x d t \rightarrow \int_{D} f_{\varepsilon}(v) \psi d x .
$$

Now (2) implies that $\int_{D} f_{\varepsilon}(v) \psi d x \leq K$ because $f_{\varepsilon}\left(U_{n}\right) \leq f\left(U_{n}\right)$. Since $f_{\varepsilon}(v) \rightarrow f(v)$ in a monotone way as $\varepsilon \rightarrow 0$, the monotone convergence theorem gives the assertion.

Theorem. If $v \in \omega\left(u_{0}\right)$ then $v$ satisfies

$$
\int_{D} v \Delta \psi d x-\lambda \int_{D} f(v) \psi d x-\int_{\partial D} \frac{\partial \psi}{\partial \nu} d S=0
$$

for any $\psi \in C^{2}(\bar{D})$ vanishing on $\partial D, \psi \geq 0$. 
Proof. We use some ideas from [Ph, LP].

Let $\left\{t_{n}\right\}$ be a sequence such that $U_{n} \rightarrow v$ in $L^{2}(D \times(0,1))$ and pointwise in $D \times(0,1)$. Let $\varphi \in C^{\infty}(\mathbb{R})$ be such that

$$
\varphi(s)= \begin{cases}s-1 & \text { for } s \geq 2, \\ 0 & \text { for } s<\frac{1}{2},\end{cases}
$$

$\varphi^{\prime}, \varphi^{\prime \prime} \geq 0$, and define $\varphi_{h}(s):=h \varphi(s / h)$ for $h>0$. Take $\rho, \psi$ as in the proof of Lemma 2 and $g(x, s)=\psi(x) \rho(s)$. Then

$$
\begin{gathered}
\int_{0}^{1} \int_{D} \varphi_{h}\left(U_{n}\right)\left(\Delta g+g_{t}\right) d x d t \rightarrow \int_{0}^{1} \int_{D} \varphi_{h}(v)\left(\Delta g+g_{t}\right) d x d t \quad \text { as } n \rightarrow \infty, \\
\int_{0}^{1} \int_{D} \varphi_{h}(v)\left(\Delta g+g_{t}\right) d x d t \rightarrow \int_{0}^{1} \int_{D} v\left(\Delta g+g_{t}\right) d x d t \quad \text { as } h \rightarrow 0,
\end{gathered}
$$

and

$$
\int_{0}^{1} \int_{D} v\left(\Delta g+g_{t}\right) d x d t-\int_{D} v \Delta \psi d x
$$

On the other hand, we obtain (cf. [Ph])

$$
\begin{aligned}
& \int_{0}^{1} \int_{D} \varphi_{h}\left(U_{n}\right)\left(\Delta g+g_{t}\right) d x d t \\
& =\lambda \int_{0}^{1} \int_{D} \varphi_{h}^{\prime}\left(U_{n}\right) U_{n}^{-\beta} g d x d t \\
& \quad+\int_{0}^{1} \int_{D} \varphi_{h}^{\prime \prime}\left(U_{n}\right)\left|\nabla U_{n}\right|^{2} g d x d t+\int_{0}^{1} \int_{D} \varphi_{h}(1) \frac{\partial g}{\partial \nu} d S d t
\end{aligned}
$$

As $n \rightarrow \infty$, we find

$$
\int_{0}^{1} \int_{D} \varphi_{h}^{\prime}\left(U_{n}\right) U_{n}^{-\beta} g d x d t \rightarrow \int_{0}^{1} \int_{D} \varphi_{h}^{\prime}(v) v^{-\beta} g d x d t=\int_{D} \varphi_{h}^{\prime}(v) v^{-\beta} \psi d x .
$$

Now $\varphi_{h}^{\prime}(v) v^{-\beta} \psi \rightarrow f(v) \psi$ pointwise, $\varphi_{n}^{\prime}(v) v^{-\beta} \psi \leq f(v) \psi$, and, by Lemma 2, $f(v) \psi$ is integrable. This implies

$$
\int_{D} \varphi_{h}^{\prime}(v) v^{-\beta} \psi d x \rightarrow \int_{D} f(v) \psi d x \quad \text { as } h \rightarrow 0 .
$$

Since $\varphi_{h}^{\prime \prime}(s) \leq\left(c_{1} / h\right) \chi_{h / 2<s<2 h}(s)$ for some constant $c_{1}>0$, we obtain

$$
\int_{0}^{1} \int_{D} \varphi_{h}^{\prime \prime}\left(U_{n}\right)\left|\nabla U_{n}\right|^{2} g d x d t \leq \frac{c_{1}}{h} \int_{0}^{1} \int_{D} \chi_{h / 2<U_{n}<2 h}\left|\nabla U_{n}\right|^{2} d x d t .
$$

From [Ph, Lemma 5] we get

$$
\left|\nabla U_{n}\right|^{2} \leq \frac{2}{1-\beta} U_{n}^{1-\beta}+M U_{n} \quad \text { for some } M>0 .
$$

This implies that

$$
\frac{c_{1}}{h} \int_{0}^{1} \int_{D} \chi_{h / 2<U_{n}<2 h}\left|\nabla U_{n}\right|^{2} g d x d t \leq c_{2} \int_{0}^{1} \int_{D}\left(U_{n}^{-\beta}+c_{3}\right) \chi_{h / 2<U_{n}<2 h} g d x d t .
$$


As $n \rightarrow \infty$, the right-hand side approaches

$$
c_{2} \int_{0}^{1} \int_{D}\left(v^{-\beta}+c_{3}\right) \chi_{h / 2<v<2 h} g d x d t,
$$

and by Lemma 2 this converges to zero as $h \rightarrow 0$.

Corollary. If $D$ is a ball, $\lambda$ is sufficiently small, and $u_{0}$ is radial, then there is a $t_{0} \geq 0$ such that $u\left(x, t ; u_{0}\right)>0$ for $x \in \bar{D}, t>t_{0}$.

Proof. If $D$ is a ball and $\lambda$ is sufficiently small, it is known that in the class of radial functions there is a unique weak stationary solution $v$, which is positive and hence classical [JL, BN]. The theorem implies then that $u$ must become positive in finite time, because it converges to $v$ uniformly as $t \rightarrow \infty$.

\section{REFERENCES}

[A] R. Aris, The mathematical theory of diffusion and reaction, Clarendon Press, Oxford, 1975.

[BB] C. Bandle and C. M. Brauner, Singular perturbation method in a parabolic problem with free boundary, Proc. BAIL IVth Conf. Novosibirsk (S. K. Godunov et al., eds.), Boole Press, Dublin, 1987, pp. 7-14.

[BN] C. M. Brauner and B. Nicolaenko, On nonlinear eigenvalue problems which extend into free boundaries problems, Proc. of Conference on Bifurcation and Nonlinear Eigenvalue Problems, Lecture Notes in Math., vol. 782, Springer, Berlin, 1980, pp. 61-100.

[DL] K. Deng and H. A. Levine, On the blow up of $u_{t}$ at quenching, Proc. Amer. Math. Soc. 106 (1989), 1049-1056.

[D] J. I. Diaz, Nonlinear partial differential equations and free boundaries, Vol. I, Elliptic Equations, Pitman Res. Notes Math. Ser., vol. 106, Longman Sci. Tech., Harlow, 1985.

[FHQ] M. Fila, J. Hulshof, and P. Quittner, The quenching problem on the $N$-dimensional ball, Nonlinear Diffusion Equations and their Equilibrium States 3 (N. G. Lloyd et al., eds.), Birkhäuser, Boston, 1992, pp. 183-196.

[FK] M. Fila and B. Kawohl, Asymptotic analysis of quenching problems, Rocky Mountain J. Math. 22 (1992), 563-577.

[JL] D. D. Joseph and T. S. Lundgren, Quasilinear Dirichlet problems driven by positive sources, Arch. Rational Mech. Anal. 49 (1973), 241-269.

[LP] M. Langlais and D. Phillips, Stabilization of solutions of nonlinear and degenerate evolution equations, Nonlinear Anal. 9 (1985), 321-333.

[L] H. A. Levine, Quenching, nonquenching and beyond quenching for solutions of some parabolic equations, Ann. Mat. Pura Appl. 155 (1989), 243-290.

[Ph] D. Phillips, Existence of solutions of quenching problems, Applicable Anal. 24 (1987), 253264.

(M. Fila and H. A. Levine) Department of Mathematics, Iowa State University, Ames, IowA 50011

E-mail address, H. A. Levine: S2.HAL@ISUMVS.BITNET

(J. L. Vazquez) Department of Mathematics, Universidad Autonoma de Madrid, 28049 MADRID, SPAIN

E-mail address: JVAZQUEZ@vm1.sdi.uam.es 